\title{
Über Cer-Magnesiumlegierungen.
}

\author{
Von Rudolf Vogel.
}

Mit 1 Figur im Text und 3 Tafeln.

Nach Muthmann und Beck ${ }^{1}$, welche zuerst eine Cer-Magnesiumlegierung herstellten, erfolgt die Vereinigung dieser beiden Metalle beim Zusammenschmelzen unter Absorption von Wärme. Diese Beobachtung weist darauf hin, da $B$ die Beziehungen des Cers zum Magnesium ganz andere sind als zu den Elementen $\mathrm{Al}, \mathrm{Si}$, Sn, $\mathrm{Pb}, \mathrm{Bi}$, mit denen es sich, wie meine früheren Versuche ergeben haben ${ }^{2}$, ausnahmslos unter ungewöhnlich großer Wärmeabgabe vereinigt. Die nachfolgende Untersuchung über das Zustandsdiagramm der Cer-Magnesiumlegierungen hat diese Vermutung in der Tat bestätigt.

Bei der Darstellung der Cer-Magnesiumlegierungen stellte sich heraus, daß sämtliche Legierungen schon bei $800^{\circ}$ vollständig flüssig sind, ein Umstand, der für das Arbeiten mit zwei bei höheren Temperaturen so außerordentlich reaktionsfähigen Metallen wie Cer und Magnesium sehr günstig ist. Die Legierungen konnten daher in Kohlegefäßen erhitzt werden, ohne daß eine Belästigung durch die Bildung von Cerkarbid zu befürchten war, ferner wurde das Thermoelement und dessen Schutzrohr durch Kohlenoxyd und die Schmelze nur wenig angegriffen und schließlich war die Möglichkeit vorhanden, einen Eisenrührer zu verwenden, welcher aus einem unten zu einer horizontalen Scheibe aufgewundenen Eisendraht bestand. Die Anwendung eines wirksamen Rührers erwies sich als unbedingt erforderlich, um Legierungen von einheitlicher Konzentration zu erzielen, da selbst bei etwa $900^{\circ}$ die Vereinigung der geschmolzenen Metalle zu einer homogenen Flüssigkeit scheinbar nur träge verläuft, so daß die Metalle, nachdem sie geschmolzen sind, ihrem Bestreben, sich ihren sehr verschiedenen spezifischen

1 Lieb. Ann. 331, 46-57.

2 Z. anorg. Chem. 72, 319; 75, 41; 84, 323. 
Gewichten entsprechend übereinander zu schichten, folgen können, während sich ihre Durchmischung erst teilweise vollzogen hat. Daß die Inhomogenität der Reguli auch hier, wie bei den Cer-Aluminiumlegierungen nicht allein in dem Untersinken oder Aufsteigen von Kristallen in einer homogenen Schmelze von anderem spezifischen Gewicht, sondern hauptsächlich in primären Konzentrationsdifferenzen innerhalb der Schmelze ihren Grund hat, folgt aus der Struktur solcher inhomogener Reguli. Das leichtere Magnesium im Schmelzrohr zu unterst, das schwerere Cer zu oberst zu legen, erwies sich zur Vermeidung dieses Übelstandes als gänzlich unzureichend und selbst durch Rühren glückte es nicht immer, die völlige Durchmischung der Schmelze herbeizuführen. In solchen Fällen wurden schließlich immer, wie auch im Falle der Cer-Aluminiumlegierungen, durch wiederholtes Schmelzen der umgekehrt in das Schmelzrohr eingeführten Reguli, Legierungen von einheitlicher Konzentration erhalten. Nur auf solche beziehen sich die nachfolgenden Messungen. Der erwähnte Übelstand schlechter Mischbarkeit ist im vorliegenden Falle noch störender als bei den Cer-Aluminiumlegierungen, wo die Bildung einheitlicher Schmelzen durch die energische Reaktion dieser beiden Elemente miteinander gefördert wird und die Inhomogenität der Reguli sich nur auf ein kleines Konzentrationsintervall $(0-27$ Gew. $0 \%$ Al $)$ beschränkt, während er sich infolge der allgemeinen Trägheit, mit der sich Cer und Magnesium vereinigen, auf alle Konzentrationen erstreckt. Die Geschwindigkeit der Vereinigung von Cer und Magnesium würde zweifellos wachsen, wenn man die Temperatur der geschmolzenen Metalle erheblich über $900^{\circ}$ hinaus steigerte. Hieran ist man aber behindert durch den rasch anwachsenden Dampfdruck des Magnesiums (Sp. bei ca. $1100^{\circ}$ ) und ferner durch die Einwirkung der Schmelze auf den Eisenrührer. Um kontrollieren zu können, ob eine solche stattgefunden hatte, wurde die Schliffläche an jedem Regulus zum Zwecke der mikroskopischen Untersuchung seiner Struktur immer so gelegt, daß der Rührer mit durchschnitten wurde. War die Legierung wesentlich über $900^{\circ}$ erhitzt worden, so sah man deutlich kleine von dem Eisendraht ausgehende und in der Masse der Legierung verstreute Kriställchen. Unter diesen Umständen wurde jeder Regulus zunächst mikroskopisch auf seine Homogenität geprüft, falls erforderlich umgeschmolzen und die Ablühlungskurve von neuem bestimmt. Die Herstellung dex Legierungen im Kohlerohr geht nach dem früher be- 
schriebenen ${ }^{1}$ einfachen Verfahren leicht und gefahrlos vonstatten. Die Oxydation der Metalle ist hierbei ganz unerheblich. Bringt man jedoch, etwa beim Herausziehen des Rührers aus dem Schmelzrohr, ein wenig von der flüssigen Legierung an die Luft, so entzündet sie sich und verbrennt unter lautem Zischen und Knattern zerspritzend, mit explosionsartiger Heftigkeit. Die leichte und gefahrlose Zugänglichkeit der Cer-Magnesiumlegierungen ist also vor allem dem Umstande zu danken, daß ihre Bildung, im Gegensatz zu den früher untersuchten Cerlegierungen nicht exotherm verläuft und daher ein Herausspritzen der Schmelze infolge heftiger Verdampfung von Magnesium nicht zu befürchten ist.

Für die Bestimmung der Abkühlungskurven genügten zwischen 75 und 100 Atom- $\% \mathrm{Mg}$ je $10 \mathrm{~g}$ Legierung zwischen 0 und 75 Atom- $\%$ $\mathrm{Mg}$ waren, der Undeutlichkeit der Wärmeeffekte wegen, je $20 \mathrm{~g}$ erforderlich. Die recht gute Luftbeständigkeit der Cer-Magnesiumlegierungen ermöglicht auch ein eingehendes Studium ihrer Struktur. Dieselbe ist nach dem Polieren der Schliffläche in der Hauptsache bereits ohne Ätzung infolge kleiner Helligkeits- und Farbkontraste der einzelnen Strukturelemente zu erkennen. Zahlreiche Versuche ergaben, daß mit Hilfe von $\mathrm{NH}_{4} \mathrm{Cl}, \mathrm{HNO}_{3}$ und $\mathrm{HCl}$ unter den verschiedensten Bedingungen keine guten Kontraste zwischen den einzelnen Strukturelementen hervorzubringen sind, weil sich diese in ihrem Verhalten gegenüber jenen Mitteln zum Teil wenig voneinander unterscheiden und daß teilweise sogar gänzlich irreleitende Bilder entstanden. Das geeignete Ätzmittel für die Cer-Magnesiumlegierungen besteht, wie sich schließlich herausstellte, in der Erzeugung von Anlauffarben. Zu diesem Zweck wurden die Reguli einfach an der Bunsenflamme langsam bis zum Erreichen des geeigneten Farbentones, gelb bis purpurrot, erhitgt, wobei eine Entzündung der Legierung nicht zu befürchten ist, und so die schönsten und deutlichsten Farbkontraste zwischen den verschiedenen Strukturelementen erzielt. Mit diesem Verfahren hat man bei allen CerMagnesiumlegierungen Erfolg, nur zwischen 90 und 100 Atom- $\%$ $\mathrm{Mg}$ ergibt ein etwa 30 Sekunden langes Ätzen der Schliffe durch kochendes Wasser noch bessere Resultate.

Umstehende Tabelle enthält die Versuchsresultate. Darin sind die eingewogenen Konzentrationen der Legierungen, wie beim Cer-Aluminium, wegen des großen Unterschiedes der Atomgewichte $(\mathrm{Ce}=140$,

1 Z. anorg. Chem. 72, 322. 
Tabelle.

\begin{tabular}{|c|c|c|c|c|c|c|c|c|c|c|c|c|c|}
\hline \multirow{2}{*}{\multicolumn{3}{|c|}{$\begin{array}{c}\text { Zusammensetzung } \\
\text { der Legierungen } \\
\text { in Gew. } \% \text { unter } \\
\text { Berücksichtigung } \\
\text { d. Beimengungen } \\
\text { des Cers }\end{array}$}} & \multicolumn{4}{|c|}{$\begin{array}{c}\text { Verhältnis } \mathrm{Mg}=\mathrm{Ce}, \\
\text { bezogen auf } \\
\mathrm{Mg}+\mathrm{Ce}: 100 .\end{array}$} & \multirow{3}{*}{ 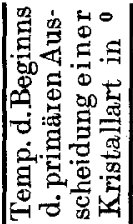 } & \multicolumn{6}{|c|}{$\begin{array}{l}\text { Temperatur u. Zeitdauer der } \\
\text { nonvarianten Gleichgewichto }\end{array}$} \\
\hline & & & \multicolumn{2}{|c|}{ Gew. .0} & \multicolumn{2}{|c|}{ Atom- $0 / 0$} & & & & & & & \\
\hline $\mathbf{M g}$ & $\mathrm{Ce}$ & Beimeng. & $\mathbf{M g}$ & $\mathrm{C}$ & $\mathrm{Mg}$ & $\mathrm{Ce}$ & & Temp & Zeit & Temp. & Zeit & Temp. & Zeit \\
\hline 0 & 93.5 & 5 & & 100 & 0 & 100 & $810^{\circ}$ & - & - & - & - & - & 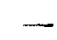 \\
\hline 1 & & & 1.0 & & 549 & 94.51 & & - & - & $632^{\circ}$ & $5^{\prime \prime}$ & 497 & $?$ \\
\hline 1,5 & & & 1.6 & 98.4 & 8.55 & 91.45 & 726 & - & - & 632 & 15 & 4 & 10 \\
\hline 2.5 & & & 2.6 & 97.4 & 13.32 & 86.68 & 680 & - & - & 632 & 35 & 497 & 15 \\
\hline 4 & & t & 4.3 & 95.7 & 20.55 & 79.45 & $\cdots$ & 632 & $40^{\prime \prime}$ & - & 一 & - & - \\
\hline 5 & 8 & .2 & 5.3 & 94.7 & 24.36 & 75.64 & 652 & imp. & & 632 & 25 & 501 & 35 \\
\hline 6 & 87.9 & 6.1 & 6.4 & 93.6 & 28.42 & 71.76 & 687 & - & ) & 625 & 15 & $4 !$ & 25 \\
\hline 7.5 & 8 & 6 & 7.9 & 92.1 & 33.05 & 66.95 & & - & - & 632 & 10 & & 25 \\
\hline 10 & & & 10.6 & 89.4 & 40.55 & 59.45 & 26 & - & - & 632 & 5 & 4 & 5 \\
\hline & & & 11.5 & 88.5 & 49 & 57.20 & 30 & $\ldots$ & - & 631 & $<5$ & 4 & $<5$ \\
\hline 12.5 & 8 & 5 & 13.2 & 86.8 & 46.67 & 53.33 & 736 & - & $=$ & $(618)$ & - & $(502)$ & - \\
\hline 14 & 80.4 & 5.6 & 14.8 & 85.2 & 50.00 & 50.00 & $\cdots$ & 738 & 70 & - & 一 & - & $\ldots$ \\
\hline 15 & 79.5 & 5.5 & 15.8 & 84.2 & 51.93 & 48.07 & 713 & imp.d & 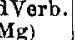 & - & 一 & - & - \\
\hline 16 & 78.6 & 5.4 & 16.9 & 83. & 53. & 46.05 & 706 & - & - & - & - & - & - \\
\hline 18 & 7 & & 19.0 & 81. & 50.48 & 42. & 726 & - & - & - & - & - & - \\
\hline 20 & & & 21.0 & 79. & & 39. & 734 & - & 一 & 716 & 15 & - & - \\
\hline 2 & & & 2 & 75. & & 35. & 753 & $\ldots$ & 一 & (717) & - & - & 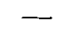 \\
\hline 30 & 6 & 4.5 & 31.4 & 68.6 & 72.50 & 27.50 & 779 & - & - & 722 & 5 & - & - \\
\hline 32 & 63.6 & 4.4 & 33.4 & 66.6 & 74 & 25.73 & & 780 & 55 & - & 一 & - & - \\
\hline 36 & 59.9 & 4.1 & 37.5 & 62.5 & 77.55 & 22.45 & 777 & Ce. & verb. & 622 & 5 & - & $\longrightarrow$ \\
\hline 40 & 56.1 & 3.9 & 41.6 & 58.4 & 0 & 19. & 771 & - & 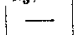 & 622 & 5 & - & 一 \\
\hline 50 & & & & 48 & & 13. & & - & - & 622 & 20 & 585 & $<5$ \\
\hline 5 & & & & 42.5 & & 11 & & - & - & 617 & 40 & 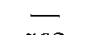 & - \\
\hline 5 & & & & 40 & & 10 & & - & $\cdots$ & 627 & 50 & & $<5$ \\
\hline 60 & & & & 38.4 & 9 & & 66 & - & $\ldots$ & 622 & 40 & & $<5$ \\
\hline 64 & & & & 34. & & & & - & - & -- & - & 57 & 15 \\
\hline 68 & & & & 31.0 & & 7. & & - & - & - & $\ldots$ & & 40 \\
\hline 70 & & & & 28.7 & 93. & 6.52 & 60 & - & - & - & - & 5 & 50 \\
\hline 75 & 2 & & 76.2 & 23.8 & & 5. & G & - & - & 一 & - & 5 & 50 \\
\hline 80 & 18 & & & 19.0 & 96. & 3. 0 & & - & - & - & - & 5 & 15 \\
\hline 90 & & 0. & & 9 & 98 & 1.77 & & - & - & - & - & 58 & 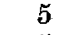 \\
\hline 97 & 2.8 & 0.2 & & 2.9 & & 0.5 & 654 & 一- & 一 & 一 & - & 585 & $<5$ \\
\hline 100 & 0 & 0 & 00.0 & 0 & 100.00 & 0 & & 661 & 100 & - & - & - & \\
\hline
\end{tabular}

$\mathrm{Mg}=24$ ) in bezug auf die Beimengungen des Cers korrigiert ${ }^{1}$. Aus diesen Resultaten folgt das Zustandsdiagramm der Cer-Magnesiumlegierungen (Fig. 1). Die Existenz von drei chemischen Verbindungen zwischen Cer und Magnesium, denen bzw. die Formeln CeMg, $\mathrm{CeMg}_{3}$ und $\mathrm{CeMg}_{9}$ zukommen, ist aus dem Zustandsdiagramm ohne weiteres ersichtlich. Außerdem bildet sich noch eine vierte Verbindung, welcher die Formel $\mathrm{Ce}_{4} \mathrm{Mg}$ zukommen muß.

1 Vgl. Z. anorg. Chem. 75, 46. 
1. Legierungen mit $0-50$ Atom $-\%$ Mg.

Mißt man bei der graphischen Darstellung der thermischen Versuchsresultate zwischen 0 und $50 \mathrm{Atom}-\% \mathrm{Mg}$ die Konzentration der Legierungen nicht, wie in Fig. 1, in Atom- $\%$, sondern, wie es bei der Ausarbeitung des Zustandsdiagramms geschah, in Ge-

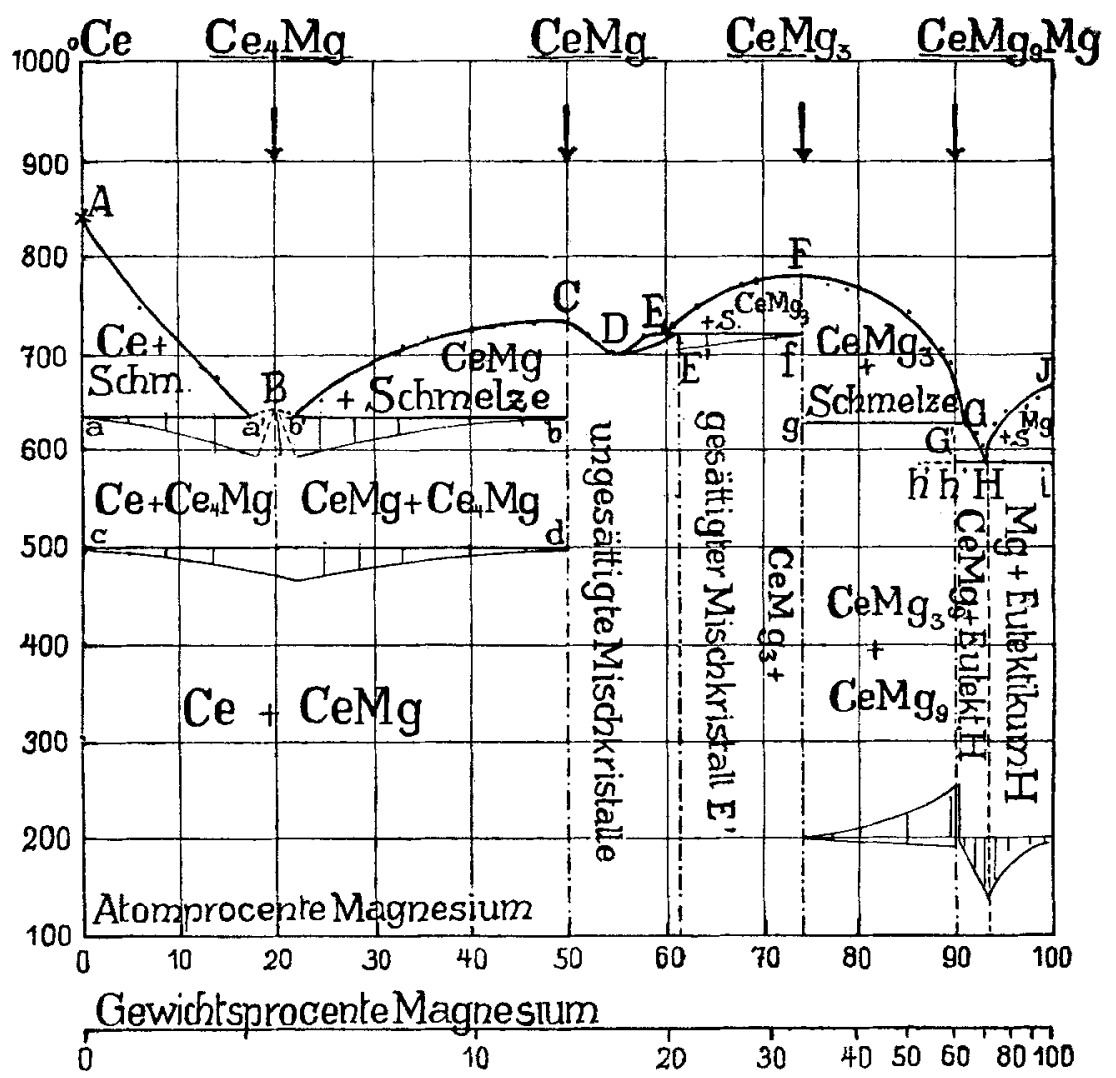

Fig. 1. Zustandsdiagramm der Cer-Magnesiumlegierungen.

wichts- $\%$, so drängen sich die ermittelten Temperaturpunkte auf den engen Konzentrationsbereich von 14 Gewichts- $\%$ zusammen und demgemäß rücken die beiden Kurvenäste $A a^{\prime}$ und $C b^{\prime}$, auf denen sich Cer bzw. die Verbindung $\mathrm{Ce} M g$ primär ausscheidet, einander so nahe, daß sie sich in einem gemeinsamen Punkt zu schneiden scheinen. Auf den Abkühlungskurven dieser Gruppe von Legierungen finden sich nun außer den primären Knickpuniten, welche die beiden Kurven $A a^{\prime}$ und $C b^{\prime}$ ergeben, durchweg noch 
zwei Haltepunkte, der exste bei rund $632^{\circ}$, der zweite bei $497^{\circ}$. Diesen Haltepunkten entsprechen im Diagramm die Horizontalen $a b$ bzw. cd. Das Maximum der Zeitdauer, welches sich wegen der Kleinheit dieser Wärmeeffekte nicht mit völliger Sicherheit ermitteln ließ, wurde für $a b$ bei $20 \mathrm{Atom}-\% \mathrm{Mg}$, für $c d$ in der Nähe dieser Konzentration, bei etwa 22 Atom- $\% \mathrm{Mg}$ gefunden. Auf Grund des thermischen Befundes hätte man also zu schließen, daß der erste Haltepunkt bei $632^{\circ}$ durch die Kristallisation eines Eutektikums aus Cer und der Verbindung $\mathrm{Ce} \mathrm{Mg}$, der zweite bei $497^{\circ}$ durch eine Reaktion im festen Zustande, bei welcher sich aus den Kristallarten $\mathrm{Ce}$ und $\mathrm{CeMg}$ eine neue Kristallart von anderer $\mathrm{Zu}$ sammensetzung bildet, hervorgerufen wird. Aus der Struktur der Legierungen folgt aber, daß diese nächstliegende Deutung der thermisch ermittelten Zustandsänderungen im vorliegenden Falle nicht zutreffen kann. Das eingehende Studium der mikroskopischen Struktur führt nämlich zu folgendem Resultat. In den Cer-reichsten Legierungen mit 5.49, 8.55 und $13.32 \mathrm{Atom}-\%(=1,1.5$ und 2.5 Gewichts- $\% \mathrm{Mg}$ ), deren Strukturbild infolge der sich hier geltend machenden Beimengungen des Cers recht kompliziert ist, kann man mit Sicherheit ein Strukturelement feststellen, das primär ausgeschiedenes Cer sein muß. Unter den übrigen Strukturelementen sind vor allem zahlreiche kleine helle Pünktchen und Lamellen charakteristisch. Dieselben sind auch zwischen 20 und $50 \mathrm{Atom-} \% \mathrm{Mg}$ zu beobachten, wo man sie in einer dunklen Grundmasse, welche die dort primär ausgeschiedenen Kristallite $\mathrm{Ce} M g$ umgibt, verstreut findet und leicht erkennen kann, daß sie aus dieser Verbindung bestehen. Von dieser Struktur sind in Fig. 2, 3, 4 (Tafel XIV) Proben wiedergegeben. Wie man sieht, ist überall dieselbe, helle Kristallart, nämlich die Verbindung CeMg, primär ausgeschieden, deren Menge in dem Maße zunimmt, als der wachsende Mg-Gehalt von 29.36, 33.05 und 46.67 Atom- $\%$ der Zusammensetzung der reinen Verbindung näher kommt. Die in der dunklen Grundmasse verteilten kleinen hellen Partikel CeMg sind besonders in Fig. 2 (Tafel XIV) deutlich erkennbar. Entscheidend für die Deutung der thermisch beobachteten Zustandsänderungen ist nun das Strukturbild einer Legierung mit 20.55 Atom- $\% \mathrm{Mg}$, welche sehr nahe dem gemeinsamen Schnittpunkte der Kurvenäste $A a^{\prime}$ und $C b^{\prime}$ entsprechen würde. Man sieht hier (Fig. 1, Tafel XIV) eine in überwiegender Menge ausgeschiedene Kristallart in Gestalt dunkler verwasehener Dreiund Vierecke. Diese Kristallart ist weder mit Cer noch mit der 


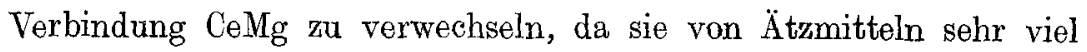
stärker als die Verbindung CeMg, dagegen schwächer als Cer selbst angegriffen wird, was auf eine zwischen $\mathrm{Cer}$ und $\mathrm{CeMg}$ liegende Zusammensetzung hinweist. Außerdem unterscheidet sie sich auch durch ihre offenbar würfelartige Form von diesen beiden Kristallarten, von denen das Cer sich nach meinen bisherigen Beobachtungen auf den Schlifflächen meistens in Form von Sechsecken und die Verbindung CeMg ebenfalls in ganz andersgearteten Gebilden darstellt. Vor allem aber zeigt die fragliche Kristallart eine deutliche und charakteristische Zerfallsstruktur. Träfe die erwähnte Auffassung zu, wonach die Schmelze $B$ bei der Temperatur von $a b$ eutektisch kristallisierte, und darauf im festen Zustande bei der Temperatur von $c d$ aus Cer und CeMg sich eine neue Verbindung bildete, so müßten sich in den Legierungen zwischen $0-50$ Atom- $\%$ $\mathrm{Mg}$, vor allem aber bei 20 Atom- $\%$ Reste dieses Eutektikums finden, da Reaktionen, bei denen auf Kosten einer oder mehrerer fester Phasen eine neue feste Phase von anderer Zusammensetzung entsteht, ja in der Regel nicht zu Ende verlaufen, falls man nicht für ganz besonders langsame Abkühlung Sorge trägt. Andeutungen für eine ursprünglich eutektische Kristallisation von $\mathrm{Ce}$ mit $\mathrm{Ce} \mathrm{Mg}$ waren jedoch in der Struktur der Reguli nirgends zu finden. Ferner würde aber auch die Zerfallsstruktur der fraglichen Kristallart mit den Aussagen der Abkühlungskurven nicht in Einklang zu bringen sein, weil dieselben alsdann außer den beiden beobachteten noch einen dritten Haltepunkt aufweisen müßten. Ein solcher wurde aber bis auf $50^{\circ}$ abwärts nicht beobachtet.

Die Tatsache, daß die fragliche Kristallart die typische Struktur eines im festen Zustande erfolgten Zerfalles in zwei andere Kristallarten von verschiedener Zusammensetzung zeigt, führt vielmehr zu dem Schluß, daß es sich bei der Zustandsänderung, welche sich bei der Temperatur von $c d$ vollzieht, nicht um die Bildung einer Verbindung, sondern um den Zerfall einer schon vorhandenen Verbindung im festen Zustande handelt, welche sich bei der Temperatur von $a b$ sekundär aus der Schmelze, gemeinsam mit einer zweiten festen Phase, ausgeschieden haben muß. Zwischen den beiden Kurvenästen $A a^{\prime}$ und $C b^{\prime}$ muß dann ein, jedenfalls sehr kleines, Kurvenstück mit einem sehr flachen Maximum $B$ angenommen werden, welches die Kurve der primären Ausscheidung der Verbindung aus der Schmelze darstellt und die Kurvenäste der primären Ausscheidung von Cer und $\mathrm{CeMg} A a^{\prime}$ bzw. $C b^{\prime}$ in je einem 
eutektischen Punkte $a^{\prime}$ bzw. $b^{\prime}$ schneidet. In der Tat zeigt sich, sobald man im Diagramm die Konzentration in Atom- $\%$ mißt, da $B$ sich die Kurvenäste $A a^{\prime}$ und $C b^{\prime}$ nicht genau bei der Temperatur von $a b$ schneiden, so daß die aus der Struktur gefolgerte Notwendigkeit, eines kleinen Kurvenstückes $a^{\prime} C b^{\prime}$ sich auch aus den thermischen Daten ergibt. Um die Existenz dieses kleinen Kurvenstückes mit Hilfe von Ablzühlungskurven experimentell nachzuweisen, würden äußerst genaue Messungen erforderlich sein, da das Maximum jedenfalls sehr flach verläuft und sich nur über das kleine Konzentrationsintervall von $5 \mathrm{Atom}-\%=1 \mathrm{Gew.} \%$ erstreckt. Aus den erhaltenen thermischen Versuchsdaten sind jedenfalls die kleinen Temperaturunterschiede, welche zwischen dem Maximum $B$ und den beiderseits angrenzenden eutektischen Horizontalen bestehen müssen, nicht mit Sicherheit zu erkennen.

Mit diesen Verhältnissein steht nun das Aussehen der Struktur vollkommen im Einklang. Der Regulus mit 20.55 Atom- $\% \mathrm{Mg}$, welcher angenähert dem Punkt $B$ entspricht, muß, abgesehen von sehr wenig sekundür ausgeschiedenem Cer, nahezu ganz aus primär ausgeschiedenen Kristallen der Verbindung bestehen, welche diesem Punkt entspricht, wie Fig.1 (Tafel XIV) es zeigt. Die beiden Eutektika $a^{\prime}$ und $b^{\prime}$ werden keine typisch eutektische Struktur haben, weil sie fast ganz aus der einen Komponente, nämlich der Verbindung bestehen. Diese muß also in allen untersuchten Reguli zwischen 0 und $50 \mathrm{Atom}-\% \mathrm{Mg}$, ausgenommen den Regulus mit $20.55 \mathrm{Atom-} \%$, die sekundär ausgeschiedene Grundmasse bilden und diese Grundmasse muß dieselbe Zerfallsstruktur aufweisen, wie die primär ausgeschiedenen Kristallite bei 20.55 Atom- $\%$ (Fig. 1, Tafel XIV. Aus den Photogrammen 1-4 ist zu ersehen, daß dies in der Tat der Fall ist. In Fig. 2, 3 und 4 ist überall die gleiche dunkle Grundmasse und ihr teilweiser Zerfall unter Ausscheidung heller, zum Teil orientierter Lamellen CeMg (besonders in Fig. 2) deutlich zu erkennen. In Fig. 1 haben sich die hellen Lamellen besonders an den Peripherien der ursprünglichen Kristallite angereichert. Die eine Zerfallskomponente, nämlich die hellen Lamellen, ist, wie schon bemerkt, leicht als die Verbindung $\mathrm{Ce}_{\Theta g} \mathrm{zu}$ erkennen, während die dunkle Grundmasse nach Vollendung des Zerfalles aus Cer bestehen würde.

Die Zusammensetzung der Verbindung ergibt sich 1. aus der Lage des Punktes $B$ bei etwa 20 Atom- $\% \mathrm{Mg}$. 
2. Aus der Lage des Maximums der Zeitdauer bei der sekundären Ausscheidung der Verbindung. Da die beiden Punkte $a^{\prime}$ und $b^{\prime}$, welche die Konzentration der Verbindung zwischen sich einschließen, einander sehr nahe liegen, die Eutektika $a^{\prime}$ und $b^{\prime}$ also fast ganz aus der Verbindung bestehen, so nimmt die Zeitdauer ihrer Kristallisation von beiden Seiten her in der Richtung auf den Punkt $B$ bis $a^{\prime}$ bzw. $b^{\prime}$ zu und erreicht, wenn man sie über diese beiden Punkte hinaus extrapoliert, bei $20 \mathrm{Atom-} \% \mathrm{Mg}$ ihr Maximum.

3. Die Zeitdauer des Zerfalles der Verbindung bej der Temperatur von $c d$ erreicht ebenfalls in der Nähe von $20 \mathrm{Atom}-\% \mathrm{Mg}$ ihr Maximum. Die letztere Konzentration ist jedoch weniger sicher bestimmt wegen der unregelmäßigen Haltezeit der betreffenden Haltepunkte. Möglicherweise erklärt sich diese Unregelmäßigkeit aus dem Einflu $B$ der Beimengungen des Cers auf die Realstion, ähnlich dem bekannten Einfluß von fremden Stoffen auf den Vollzug polymorpher Umwandlungen ${ }^{1}$. Versuche, die Reaktion durch Erhitzen der Reguli bis in das Zustandsfeld der Verbindung und darauffolgendes Abschrecken zu überspringen, hatten keine entscheidende Veränderung der Struktur zur Folge.

Aus den angeführten Daten ergibt sich für die dem Punkt $B$ entsprechende Verbindung die Formel $\mathrm{Ce}_{4} \mathrm{Mg}$. Diese wenig beständige Verbindung ist nur innerhalb des engen Temperaturbereiches von $135^{\circ}$, nämlich von $497-632^{\circ}$ stabil. Ihr Zerfall bei $497^{\circ}$ vollzieht sich gemäß der Gleichung

$$
\mathrm{Ce}_{4} \mathrm{Mg} \rightarrow \mathrm{CeMg}+\mathrm{Ce} .
$$

Verläuft diese Reaktion zu Ende, so bestehen demnach die Legierungen von $0-50$ Atom- $\% \mathrm{Mg}$ bei Zimmertemperatur aus Cer und der Verbindung CeMg.

\section{Legierungen mit 50-70 Atom- $\%$ Mg.}

Die Endglieder dieser Gruppe sind die oben erwähnte Verbindung $\mathrm{CeMg}$ und eine weitere Verbindung von der Formel $\mathrm{CeMg}_{3}$. Die Formel der Verbindung CeMg ist durch folgende Daten sicher gestellt.

1. Das Maximum $C$ liegt bei 50 Atom- $\%$ Mg.

1 Z. B. der Einfluß von Pinksalz auf die Umwandlung von weißem Zinn in graues. CoHen, Z. phys. Chem. 30, 614. 
2. Die Zeitdauer der sekundären Kristallisation bei der Temperatur von $b b^{\prime}$ verschwindet bei 50 Atom- $\% \mathrm{Mg}$.

3. Auch die Zeitdauer des Zerfalles der Verbindung CeMg bei $497^{\circ}(c d)$ verschwindet bei nahezu derselben Konzentration, nämlich bei $49 \mathrm{Atom-} / \mathrm{Mg}$.

4. Die Abküuhlungskurve einer Legierung mit $50 \mathrm{Atom-} \% \mathrm{Mg}$ zeigt einen dem Schmelzpunkt der Verbindung bei $738^{\circ}$ entsprechenden ausgeprägten Haltepunkt und weiter keinen thermischen Effekt.

5. Der Regulus mit 50 Atom- $\% / \mathrm{Mg}$ besteht, wie die mikroskopische Untersuchung seiner Struktur ergibt, ganz aus Kristallen CeMg, welche wir bereits in den Photogrammen 2, 3 und 4 primär ausgesehieden und in wachsender Menge gesehen haben. In Fig. 5 (Tafel XIV) erkennt man deutlich die durch Anreicherung geringer Verunreinigungen sichtbar gewordenen Grenzen, wo je 3 Kristallite der Verbindung CeMg zusammenstoßen.

Wie das Diagramm zeigt, scheiden sich aus Ce-reicheren Schmelzen Kristalle der reinen Verbindung, aus Ce-ärmeren hingegen Mg-reichere Mischkristalle der Verbindung aus. Die Struktur einer solchen aus Mg-reicheren Mischkristallen bestehenden Legierung, nahe dem Minimum D, ist in Fig. 6 (Tafel XIV) wiedergegeben. Das Strukturbild, welches dem der reinen Verbindung (Fig. 5, Tafel XIV) sehr ähnlich ist, zeigt, daß die durch dunkelgefärbte Verunreinigungen getrennten Mischkristalle in sich völlig homogen sind. Letzteres ist nach der Abkühlungskurve dieser Legierung auch zu erwarten, da die Ausscheidung der Mischkristalle nicht ein deutliches Kristallisationsintervall, sondern einen mehr einem Haltepunkt ähnlichen Effekt hervorruft, die Mischkristalle also ungefähr dieselbe Zusammensetzung haben müssen, wie die Schmelze, aus der sie sich bilden. Die Kurven des Beginnes und des Endes der Kristallisation fallen also praktisch zu einer einzigen Kurve $C D$ zusammen. In Fig. 6 (Tafel XIV) fällt ferner auf, daß die Schnittflächen der Kristallite von feinen dunklen Linien durchzogen werden, welche öfters verästelt, im ganzen aber unter sich parallel und auf den einzelnen Kristalliten in verschiedener Richtung orientiert sind. Diese Liniensysteme konnten durch stärkeres Ätzen auch bei der reinen Verbindung (CeMg) erzeugt werden. Um Gleitlinien handelt es sich hier wohl nicht, jedoch weist ihre Orientierung darauf hin, daß sie zu dem innern Aufbau der Kristalle in Beziehung stehen müssen. Vielleicht hat man darin die Spuren einer polymorphen Umwandlung der Ver- 
bindung $\mathrm{CeMg}$ zu erblicken, welche - jedenfalls zwischen 738 und $300^{\circ}$ - keinen merklichen Wärmeeffekt hervorruft.

Bei 54 Atom- $\% \mathrm{Mg}$ hat der Kurvenzug $C D E$ ein Temperaturminimum $D$. Der einzige und sehr ausgeprägte thermische Effekt bei $706^{\circ}$ auf der Abkühlungskurve einer. Legierung mit 53.95 Atom- $\%$ $\mathrm{Mg}$, welchen man wohl als Haltepunkt ansprechen darf, liegt nämlich tiefer als die der benachbarten Legierungen. Vor allem folgt aber aus der Struktur dieses Regulus, da $B$ der Punkt $D$ nicht etwa ein eutektischer ist, sondern in der Tat dem niedrigst schmelzenden Glied einer ununterbrochenen Mischkristallreihe $C E^{\prime}$ entspricht, denn diese Legierung zeigt ebensowenig eine eutektische Struktur wie die übrigen zwischen 50 und $62 \mathrm{~A}$ tom- $\% \mathrm{Mg}$, man konnte sich vielmehr überzeugen, da $B$ diese Legierungen, abgesehen von hin und wieder bemerkbaren kleinen fremden Beimengungen, nur aus einer Kristallart bestanden. Das Mg-reichste Endglied der Mischungsreihe ist das gesättigte Mischkristall $E^{\prime}$ mit $62 \mathrm{Atom-} \% \mathrm{Mg}$. Der Punkt $E^{\prime}$ liegt dem Punkt $E$ sehr nahe, fällt aber nicht mit ihm zusammen, was sich indirekt auf mikroskopischem Wege leicht nachweisen läßt. In Fig. 7 (Tafel XV), welche die Struktur einer Legierung mit 60.5 Atom- $\% \mathrm{Mg}$ wiedergibt, sieht man nämlich, da $\beta$ die kleinen rundlichen Kristallite, aus denen die Legierung in der Hauptsache besteht und die man wohl als den gesättigten Mischkristall $E^{\prime}$ anzusprechen hat, hier und da kleine helle Einschlüsse, jedenfalls Relikte der Verbindung $\mathrm{CeMg}_{3}$ aufweisen, während die rundlichen Kristallite selbst noch von einer etwas dunkleren Grundmasse umgeben sind. Das Vorhandensein umhüllter Relikte einer dritten Kristallart deutet aber stets auf eine nicht zu Ende verlaufene Reaktion, bei der sich eine neue Kristallart auf Kosten einer bereits vorhandenen von anderer Zusammensetzung gebildet hat. Fielen die Punkte $E$ und $E^{\prime}$ zusammen, so könnte die Legierung nur aus zwei Kristallarten, der primär ausgeschiedenen Verbindung $\mathrm{CeMg}_{3}$ und dem sekundär ausgeschiedenen gesättigten Mischkristall $E^{\prime}$, welcher beim Erhitzen zu einer homogenen Flüssigkeit von gleicher Zusammensetzung schmelzen würde, bestehen. Der gesättigte Mischkristall $E^{\prime}$ kann also nicht auf diese Weise entstanden sein, sondern muß sich gemäß der Gleichung:

$$
\mathrm{CeMg}_{3}+\text { Schmelze } E \rightleftarrows E^{\prime}
$$

gebildet haben.

Aus Schmelzen mit 60-75 Atom- $\% \mathrm{Mg}$ scheidet sich primär 
die Verbindung $\mathrm{CeMg}_{3}$ aus, von welcher sich, wie schon erwähnt, ein sehr kleiner Teil bei der Temperatur von $E f$ gemäß obiger Gleichung zur Bildung des gesättigten Mischkristalls verbraucht wird. Fig. 8 und 9 (Tafel XV) zeigen die hellen primär ausgeschiedenen Kristalle $\mathrm{CeMg}_{3}$, Fig. 8 (Tafel XV) (64.76 Atom-\% $\% \mathrm{Mg}$ ) in kleinerer, Fig. 9 (Tafel XV) (72.50 Atom- $\%$ Mg), gemäß der größeren Annäherung an die Konzentration der reinen Verbindung, in entsprechend größerer Menge. Besonders in Fig. 9 (Tafel XV) sieht man wohlausgebildete, mehrfach zu Zwillingen verwachsene und augenscheinlich sehr nahe würfelförmoge Kristalle. Die dunklere Grundmasse läBt besonders in Fig. 8 (Tafel XV) eine deutlich polyedrische Struktur erkennen und besteht in beiden Fällen aus dem gesättigten Mischkristall $E^{\prime}$. Bei der direkten mikroskopischen Betrachtung bieten die Strukturbilder dieser und der noch etwas $\mathrm{Mg}$ reicheren Legierungen, wenn man sie durch Anlauffarben hervorruft, einen prächtigen Anblick. So zeigten sich die in Fig.9 (Tafel XV) abgebildeten Kristalle nach dem Anlassen in hellglänzendem Ockergelb, die Grundmasse in leuchtendem Blau.

Die Gründe, aus denen sich die Formel der Verbindung $\mathrm{CeMg}_{3}$ ergibt, sind die folgenden:

1. hat die Kurve der primären Ausscheidung $E F G$ ein wohlausgebildetes Maximum $F$ bei 75 Atom- $\% \mathrm{Mg}$;

2. verschwindet die Zeitdauer der Reaktion bei $F f$ gemäß Gleichung (2) bei 75 Atom- $\% \mathrm{Mg}$;

3. bei nahezu derselben Konzentration, nämlich bei 76 Atom- $\%$ $\mathrm{Mg}$ wird auch die Zeitdauer der im folgenden zu besprechenden Umwandlung bei $g G$ gemäß Gleichung (3) gleich Null;

4. hat die Abkühlungskurve einer Legierung mit 74.27 Atom- $\%$ $\mathrm{Mg}$ bei $780^{\circ}$ einen Haltepunkt, während weitere, von den Umwandlungen bei $E f$ und $G g$ herrührende thermische Effekte nicht beobachtet wurden;

5. zeigt demgemäß der Schliff dieser Legierung bei der mikroskopischen Betrachtung das typische Strukturbild eines einheitlichen Stoffes, wie aus Fig. 10 ersichtlich. Die Umrisse einzelner Polyeder der Verbindung $\mathrm{CeMg}_{3}$ sind an den teils schärfer, teils schwächer hervortretenden dunklen Trennungslinien deutlich erkennbar. Hieraus folgt übereinstimmend das Verhältnis von 1 Atom Cer zu 3 Atomen Magnesium im Molekül dieser Verbindung. 


\section{Legierungen mit 75-100 Atom- $\%$ Mg.}

Die primäre Ausscheidung der Verbindung $\mathrm{CeMg}_{3}$ aus $\mathrm{Mg}$-reicheren Schmelzen vollzieht sich längs des Kurvenastes FG. Wenn die Schmelze die durch den Punkt $G$ bestimmte Temperatur und Zusammensetzung erreicht hat, reagiert sie mit den primär ausgeschiedenen Kristallen $\mathrm{CeMg}_{3}$ unter Bildung einer weiteren noch $\mathrm{Mg}$ reicheren Verbindung von der überraschenden Formel $\mathrm{CeMg}_{9}$ gemäß der Gleichung:

$$
\mathrm{CeMg}_{3}+\text { Schmelze } G \rightleftarrows \mathrm{CeMg}_{9} \text {. }
$$

Ähnlich wie bei der bereits besprochenen Umwandlung nach Gleichung (2) hat auch hier die bei Wärmeentziehung neu sich bildende Kristallart $\mathrm{CeMg}_{9}\left(G^{\prime}\right)$ eine nur wenig andere Zusammensetzung wie die Schmelze $G$, aus der sie sich unter Aufzehrung einer kleinen Menge von CeMg bildet. Auch hier beweist der Umstand, daß man zwischen 85 und 90 Atom- $\% \mathrm{Mg}$ die Kristalle $\mathrm{CeMg}_{3}$ von einer Rinde aus $\mathrm{CeMg}_{9}$ umhüllt und außerdem als drittes Strukturelement das Eutektikum $H$ vorfindet (vgl. Fig. 12, Tafel XV), die Existenz einer Reaktion im Sinne obiger Gleichung

Die Existenz der Verbindung und ihre Formel $\mathrm{CeMg}_{9}$ ist durch folgende Umstände sichergestellt.

1. Die Kurve $F G$ wird in $G$ von einer zweiten Kurve $G H$, auf welcher eine andere Kristallart, nämlich $\mathrm{CeMg}_{9}$, mit der Schmelze im Gleichgewicht ist, geschnitten. Dementsprechend beobachtet man auf den Ablkühlungskurven zwischen $g$ und $G$ Haltepunkte, welche durch die Bildung dieser neuen festen Phase nach Gleichung (3) erzeugt werden.

2. Die Zeitdauer der Reaktion bei der Temperatur von $g G$ nimmt in der Richtung auf $G$ zu und erreicht bei $90 \mathrm{Atom- \%} / \mathrm{Mg}$ ihr Maximum. Die Änderung der Zeitdauer in Abhängigkeit von der Zusammensetzung der Legierungen ist in Fig. 1 weiter unten, in der Richtung nach oben, eingetragen. Demnach bildet sich bei 90 Atom- $\% \mathrm{Mg}$ die maximale Menge der Verbindung und diese Konzentration entspricht ihrer Zusammensetzung.

3. Die Haltepunkte der eutektischen Ausscheidung $H$ treten zwar infolge unvollständigen Verlaufes der Realktion (3) bis etwa $h^{\prime}$ auf. Da dieselben aber nicht dem Gleichgewichtszustande entsprechen, hat man sie bei der Extrapolation der Haltezeiten zwischen $h$ und $H$ auszuschalten und findet alsdann, daB die Zeit- 
dauer im Punkt $h$ bei $90 \%$ Null wird. Aus vorstehendem folgt für die bei der Temperatur von $g G$ sich bildende Kristallart die obenerwähnte Formel, wonach 9 Magnesiumatome mit einem Cer-Atom sich zu dem Molekül Ce $\mathrm{Mg}_{9}$ vereinigen.

Aus Schmelzen, deren Zusammensetzung zwischen $G H$ liegt, scheidet sich diese Verbindung primär aus. Die zuletzt restierende Schmelze von der Zusammensetzung $H$ kristallisiert zum Schluß zu einem Eutektikum aus $\mathrm{CeMg}_{9}$ und Magnesium, das sich primär längs $J H$ ausscheidet.

Mit den dargelegten Gleichgewichtsverhältnissen in diesem Teil des Zustandsdiagramms steht das Aussehen der Struktur der Reguli im Einklang. Fig. 11 (Tafel XV) zeigt bei $77.5 \mathrm{Atom}-\% \mathrm{Mg}$, also in der Nähe der Verbindung $\mathrm{CeNg}_{3}$ eine überwiegende Menge großer, infolge stärkeren Anlassens dunkler Kristallite dieser Verbindung, umgeben von wenig sekundär ausgeschiedener heller Grundmasse aus $\mathrm{CeMg}_{9}$. Jene erschien nach dem Anlassen dunkelpurpurrot, diese glänzend hellgelb. Verglejcht man Fig. 11 mit Fig. 9 (Tafel XV), so ist es auffallend, daß die Verbindung $\mathrm{Ce}_{3}$ bei ihrer Ausscheidung aus Mg-reicheren Schmelzen Kristallite mit mehr abgerundeten Umrissen bildet, hingegen aus Cer-reicheren Schmelzen in Form schön ausgebildeter würfelartiger Krjstalle sich ausscheidet. Nach Tammann ${ }^{1}$ hängt es von dem mit der Temperatur sich ändernden Verhältnis der Oberflächenspannung zu den Festigkeitskräften $a b$, ob sich sphäroidale oder polyedrische Begrenzungsflächen ausbilden. Da aber im vorliegenden Falle verschiedenartige Begrenzungsflächen bei nahezu denselben Temperaturen entstehen, so sind hier zwei Fälle möglich. Entweder vermag die Verbindung $\mathrm{CeNg}_{3}$ geringe Mengen von Cer im festen Zustande zu lösen und wir hätten dann in den Würfeln auf Fig. 9 und 8 einen Cer-reicheren Mischkristall der Verbindung $\mathrm{CeMg}_{3}$ vor uns, dessen scharf ausgebildete Polyederform auf seine größere Härte, welche die Oberflächenkräfte überwindet, zurückzuführen ist. Oder die abgerundeten Gebilde in Fig. 11 sind eine weichere instabile Form der Verbindung. Umgekehrt würde dann hier die Ủberwindung der geringeren Festigkeitskräfte durch die Oberflächenspannung die mehr abgerundeten Formen in Fig. 11 erklären.

1 Über die Abhängigkeit der Kristallform von der Temperatur und die Rekristallisation in Konglomeraten. Aus den Nachrichten der K. Gesellschaft der Wissenschaften zu Göttingen. Mathemat. physikal. Klasse. 1912. 
In der schon erwähnten Fig. 12, (Tafel XV) (88.64 Atom- $\% \mathrm{Mg}$ ) sieht man eine typische Umhüllungsstruktur. Wenige dunkelgeätzte Relikte der Verbindung $\mathrm{CeMg}_{3}$, deren zerfressene Ränder von der stattgehabten Reaktion (Gleichung 3) zeugen, sind hier vollständig umhüllt von der etwas helleren Masse der Verbindung $\mathrm{CeMg}_{9}$. Dieselbe erscheint stellenweise ganz deutlich in kleinere Polygone aufgeteilt und wird ihrerseits wieder umgeben von einem dritten Strukturelement, einer hellen Grundmasse, die als Magnesium, das von der eutektischen Ausscheidung $H$ herstammt, zu betrachten ist. Daß die Struktur des Eutektikums $H$ (vgl. Fig. 15, Tafel XVI) hier kaum ausgebildet ist, ist eine Erscheinung, die man bei der Ausscheidung kleiner Mengen von Eutektikum fast immer beobachtet, wenn eine der Komponenten, wie hier $\mathrm{CeMg}_{9}$, bei der eutektischen Kristallisation die führende ist ${ }^{1}$. Die Struktur einer Legierung, die mit 91.61 Atom- $\% \mathrm{Mg}$ nahe bei der Verbindung $\mathrm{Ce}_{0} \mathrm{Mg}_{9}$ liegt, und ziemlich genau dem Knickpunkt $G$ entspricht, ist in Fig. 13 abgebildet. Die Verbindung erscheint in Form großer heller parallel gelagerter Balken, zwischen denen sich wenige, hier im Gegensatz zu Fig. 12 dunkel geätzte Magnesiumkristallite eutektisch ausgeschieden haben. Dieses sowie die noch folgenden Strukturbilder wurden durch Ätzen der Schliffe unit kochendem Wasser hervorgerufen, wobei sich das Magnesium je nach der Dauer des Ätzens braun bis schwarz färbt, während die Verbindung $\mathrm{CeMg}_{9}$ nicht angegriffen wird. Fig. 14 $(92.78 \mathrm{Atom}-\% \mathrm{Mg}$ ) zeigt entsprechend weniger primär ausgeschiedene helle Kristallite $\mathrm{CeMg}_{9}$, umgeben von einer größeren Menge Eutektikum $H$, dessen Struktur hier deutlich ausgebildet ist. Das reine Eutektikum $H$ sieht man in Fig. 15. Ein Überblick über einen größeren Teil der Schliffläche als der hier abgebildete läßt deutlich einzelne Zentren erkennen, von denen die eutektische Kristallisation unter Erzeugung erst sehr feiner, dann immer gröberer, in Fig. 15 (Tafel XVI) vorwiegend im Querschnitt sichtbarer Säulen, ausgegangen ist. ${ }^{1}$ An den Rändern der einzelnen Kristallisationssysteme sind daher die Schnitte durch die Säulen dicker als im Innern, infolgedessen bilden die größeren hellen und dunklen Flecke in Fig. 15 (Tafel XVI) eine netzförmige Zeichnung, was in der Figur leider nur andeutungsweise zu erkennen ist.

Primär ausgeschiedenes und dendritisch angeordnetes Magnesium zeigt Fig. 16, Tafel XVI (96.1 Atom- $\% \mathrm{Mg})$. Es ist dunkel

${ }^{1}$ R. Voakt, Über eutektische Kristallisation, Z. anorg. Chem. 76 (1912), 425. 
geätzt, die Verbindung $\mathrm{CeMg}_{9}$ hell. Bei 98.23 Atom- $\% \mathrm{Mg}$ hat die Struktur das in Fig. 17 abgebildete Aussehen. Große tiefschwarze Magnesiumkristallite sind von einer geringen Menge des hell erscheinenden Eutektikums $H$ umgeben. Schließlich zeigt Fig. 18 (Tafel XVI) noch, wie sich Ausscheidungen des Eutektikums nach langsamer Kristallisation auf der ungeätzten Schliffläche derselben Legierung mit 98.23 Atom- $\% \mathrm{Mg}$ darstellen. In einer homogenen Grundmasse aus Magnesium erblickt man feine, zierliche Skelette, bestehend aus $\mathrm{CeMg}_{9}$, deren Orientierung nach kristallographischen Richtungen beweist, daß sie sich in der Schmelze zuerst gebildet und dadurch erst die Ausscheidung von Magnesium nach sich gezogen haben. $\mathrm{CeMg}_{9}$ ist somit bei der Ausscheidung des Eutektikurns $H$ die führende Komponente.

\section{Eigenschaften der Cer-Magnesiumlegierungen.}

Für die Eigenschaften der Cer-Magnesiumlegierungen sind, neben den Eigenschaften von Cer und Magnesium selbst, vor allem die der chemischen Verbindungen, welche unter ihmen vorkommen, maßgebend. Den Verbindungen ist folgendes eigentümlich.

1. $\mathrm{Ce}_{4} \mathrm{Mg}$ mit $20 \mathrm{Atom}-\%=4.2$ Gew.- $\%$ Magnesium schmilzt bei $632^{\circ} \mathrm{zu}$ einer homogenen Flüssigkeit, in welcher die Verbindung, nach der ganz auffallenden Flachheit und tiefen Lage des Maximums $B$ zu schließen, sehr stark dissoziiert sein muß. Kühlt man die kristallisierte Verbindung bis auf $497^{\circ} \mathrm{ab}$, so zerfällt sie unter Wärmeabgabe gemäß Gleichung (1) in Cer und die Mg-reichere Verbindung CeMg. Dieser Zerfall muß, wenn man das System lange genug einer Temperatur dicht unter $497^{\circ}$ aussetzt, ein vollständiger sein. Andrerseits bildet sich die Verbindung beim Erhitzen auf $497^{\circ}$ unter Aufnahme von Wärme. Die Verbindung $\mathrm{Ce}_{4} \mathrm{Mg}$ ist also endotherm und wir haben in ihr ein interessantes Beispiel, welches die Eigentümlichkeiten einer lockeren, unbeständigen Verbindung: endotherme Bildungsweise, tiefen Schmelzpunkt und ein sehr eng begrenztes Stabilitätsgebiet aufweist. Der Nachweis einer endothermen Cer-Magnesiumverbindung ist auBerdem bemerkenswert im Zusammenhang mit der von MuthmanN ${ }^{1}$ gemachten Beobachtung, daß die Bildung einer Cer-Magnesiumlegierung von 46.94 Gew. $-\% / 0 \mathrm{Ce}$ beim Zusammenschmelzen ebenfalls ein endothermer Vorgang ist, 
und man daher möglicherweise auch die Verbindungen $\mathrm{CeMg}$ und $\mathrm{Ce}_{\mathrm{Mg}}$, deren zwar etwas höher rückenden Schmelzpunkte doch noch verhältnismäßig recht niedrig liegen, als endotherm betrachten kann. Über die weiteren Eigenschaften der Verbindung $\mathrm{Ce}_{4} \mathrm{Mg}$ läßBt sich wenig Bestimmtes sagen, weil sie, wenn Gleichgewicht eingetreten ist, bei Zimmertemperatur vollständig in Cer und $\mathrm{CeMg}$ zerfallen ist und weil sie auch durch Abschrecken nicht anders als teilweise zerfallen, also gemischt mit den Kristallarten Cer und CeMg erhalten werden konnte. Jedenfalls erwies sich sowohl der abgeschreckte als auch der nicht abgeschreckte Regulus mit 20.55 Atom- $\%=4.3 \mathrm{Gew} .-\% \mathrm{Mg}$, welcher ungefähr ihrer Zusammensetzung entspricht, als pyrophor. Ob diese Eigenschaft insbesondere der Verbindung $\mathrm{Ce}_{4} \mathrm{Mg}$ eigentümlich ist, bleibt aus dem obigen Grunde unentschieden.

2. Die Verbindung CeMg (50 Atom- $\%=14.8$ Gew. $-0 \% \mathrm{Mg}$ ) hat ihren Schmelzpunkt bei $738^{\circ}$. Während ihrer Kristallisation findet augenscheinlich eine erhebliche Kontraktion statt, da der Regulus nach dem Erkalten der Wand des Schmelzrohrs nur lose anliegt und seine Oberfläche stark eingebeult erscheint. Die Verbindung ist sehr hart, die Härte erreicht nahezu den 5. Grad der Mossschen Skala. Die Bruchfläche des Regulus hat graurötliche Farbe und setzt sich aus kleinen, mattschimmernden Kristallflächen zusammen. Die Verbindung CeMg ist stark pyrophor, eine Eigenschaft, die aber erst bei sehr kräftigem Feilen überraschend zum Vorschein kommt. Die alsdann eintretende Verbrennung der abgetrennten Teilchen erfolgt unter äußerst heftigem Zischen und Funkensprühen, welches sich bis zu einer Flammenerscheinung von fahlgelbem Lichte steigern läßt; zugleich entwickelt sich weißlicher Oxydrauch in nicht erheblicher Menge. Kleine Stückehen der Verbindung beginnen, wenn man sie in der Bunsenflamme erhitzt, alsbald infolge Oxydation selbständig zu glühen, erhitzt man dann noch stärker, so erfolgt plötzlich eine explosionsartige Verpuffung. Diese letztere Erscheinung scheint in den Mg-reicheren Mischkristallen der Verbindung ihr Maximum zu erreichen. Kleine Körnchen dieser Legierungen von weniger als $1 / 10 \mathrm{~g}$ explodieren bisweilen mit erschreckender Heftigkeit unter lautem Knall. Diese Erscheinung, welche bei keiner der früher von mir untersuchten Cer-Legierungen beobachtet wurde, hat man wohl auf die leichte Verdampfbarkeit von Magnesium, dessen Siedepunkt ja nur wenig über $1000^{\circ}$ liegt, zurückzuführen. Möglicherweise kommt die Explosionserscheinung da- 
durch zustande, daß eine Oxydkruste ${ }^{1}$, welche sich bei der vorausgehenden äußerlichen Verbrennung um die mittlerweile flüssig gewordene Legierung gebildet hat, durch Magnesiumdampf gesprengt wird.

Aus vorstehendem ist zu folgern, daß die Oxydationsgeschwindigkeit der Verbindung CeMg erst von einer erheblich über der Zimmertemperatur liegenden Temperatur ab merklich wird, dann aber sehr schnell ansteigt. Hiermit stimmt überein, daß die Verbindung bei Zimmertemperatur an der Luft recht beständig ist. Nach monatelangem Liegen an feuchter Luft war die polierte Schliffläche kaum merklich oxydiert. Von Mineralsäuren, auch verdünnten, wird die Verbindung angegriffen, jedoch erheblich weniger als Cer.

3. $\mathrm{CeMg}_{3}\left(75\right.$ Atom- $\%=33.9$ Gew. $\%$ Mg) schmilzt bei $780^{\circ}$, ist weniger hart als $\mathrm{CeMg}$ (Härte $=4$ ) und zerbrechlicher; beim Feilen trennen sich starkglänzende Blättchen ab. Sie ist nicht pyrophor. Kleine Stückchen verbrennen beim Erhitzen unter Zischen, jedoch weniger heftig als CeMg. Dementsprechend ist die Verbindung auch an der Luft noch beständiger als $\mathrm{CeMg}$ und wird von Säuren etwas weniger angegriffen.

4. $\mathrm{CeMg}_{9}$ (90 A tom- $\%=61.02$ Gew.- $\% \mathrm{Mg}$ ) zerfällt beim Erhitzen auf $622^{\circ}$ in die Cer-reichere Verbindung $\mathrm{CeMg}_{3}$ und eine Mg-reichere Schmelze G. Die Legierung mit $91.61 \mathrm{Atom}-\% \mathrm{Mg}$, die, abgesehen von geringen Mengen des Eutektikums $H$ ganz aus Kristallen der Verbindung besteht (vgl. Fig. 13, Tafel XVI), hat die Härte 3 , ist leicht zerbrechlich und zeigt im Bruch schönen Silberglanz. Diese Verbindung besitzt die Eigentümlichkeit, im Laufe von Tagen und Wochen allmählich in kleine metallische Körnchen zu zerrieseln, welche sich auch bei langem Liegen an der Luft nicht oxydieren. Das andauernde laute Knistern, welches nach vollständiger Kristallisation der Legierung während ihrer weiteren Ablkühlung vernehmbar ist, läßt darauf schließen, daß sich innerhalb der Legierung - eine Einwirkung der Legierung auf das Schmelzrohr fand nicht statt - Volumveränderungen vollziehen, welche vielleicht einer polymorphen Umwandlung der Verbindung zuzuschreiben und daß hierin die Ursache des Zerrieselns zu erblicken ist. Gegen Oxydation und Einwirkung von Säuren ist

1 Die Bildung einer solehen harten, das Metall vollständig umhüllenden Oxydkruste wurde bei langsamem Erhitzen von Cer auf höhere Temperatur beobachtet. Z. anorg. Chem. 72, 321 . 
die Verbindung $\mathrm{Ce}_{\mathrm{Mg}}$ noch beständiger als $\mathrm{CeMg}_{3}$. Körnchen der Verbindungen verbrennen beim Erhitzen ruhig und mit glänzend weißer Flamme wie Magnesium. Von kochendem Wasser wird die Verbindung nicht zersetzt; sie ist hierin also chemisch weniger reaktionsfähig als ihre beiden Komponenten Cer und Magnesium. Sie ist von den Cer-Magnesiumverbindungen die gegen chemische Agenzien beständigste.

Betreffs der pyrophoren Eigenschaften der Legierungen im allgemeinen sei noch bemerkt, daß dieselben sich auf das Konzentrationsintervall 20 bis etwa 62 Atom- $\%=4-22$ Gew. $\%$ erstrecken. Am leichtesten und reichlichsten lassen sich Funken hervorrufen in der Gegend von etwa $40 \mathrm{Atom}-\%$ gleich ungefähr 10 Gew.- $\%$ Mg. Bei dieser Konzentration entzünden sich die abgeriebenen Teilchen sehr leicht, oft schon beim Schleifen der Reguli auf Schmirgelpapier, wodurch eine auf demselben umherhuschende Feuererscheinung hervorgerufen wird. Die größere Leichtigkeit der Verbrennung wird jedenfalls verursacht durch den gleichzeitigen Gehalt der Legierung an der sehr harten, zum Teil fein verteilten Verbindung $\mathrm{Ce} \mathrm{Mg}$ und dem weicheren, aber viel leichter oxydabeln Cer. Abgetrennte, weiche Cerpartikel und harte Körnchen der Verbindung CeMg werden sich bei ihrer gegenseitigen Reibung stark erhitzen, wodurch erst die Entzündung von Cer eingeleitet wird, die dann ihrerseits die erst bei höherer Temperatur eintretende heftige Verbrennung der Verbindung CeMg nach sich zieht. An der Luft ist eine solche Legierung mit etwa $10 \mathrm{Gew.-} \% \mathrm{Mg}$ trotz ihres Gehaltes an freiem Cer, der von dem Zerfall der Verbindung $\mathrm{Ce}_{4} \mathrm{Mg}$ herrübrt, noch recht beständig.

\section{Schluß.}

Cer und Magnesium bilden nach vorstehender Untersuchung vier chemische Verbindungen $\mathrm{Ce}_{4} \mathrm{Mg}$, $\mathrm{CeMg}, \mathrm{CeMg}_{3}, \mathrm{CeMg}_{9}$. Unter ihnen fällt die ungewöhnliche Formel $\mathrm{CeMg}_{9}$ auf, deren einziges bekanntes Analogon die Verbindung $\mathrm{KHg}_{9}$ ist, merkwürdigerweise zugleich das Endglied einer ganz ähnlichen Verbindungsreihe $\mathrm{KHg}$, $\mathrm{KHg}_{3}, \mathrm{KHg}_{9}$, Die große Verbindungsfähigkeit des Cers mit andern Elementen, für welche wir in seinem Verhalten zu Al, $\mathrm{Si}, \mathrm{Sn}, \mathrm{Pb}, \mathrm{Bi}$ typische Beispiele kennen gelernt haben, äußert sich auch gegenüber dem Magnesium. Vergleicht man aber die Cer-Magnesiumverbindungen mit den $\mathrm{Al}-$, Si-, $\mathrm{Sn}-, \mathrm{Pb}-$ und $\mathrm{Bi}-$ Verbindungen des 
Cers, so fällt sofort ein sehr bedeutsamer Unterschied auf. Während nämlich diese letzteren Verbindungen durch ihre stark exotherme Bildungsweise und ihre ungewöhnlich hohen Schmelztemperaturen als sehr feste Verbindungen gekennzeichnet sind, zeigen hingegen die Cer-Magnesiumverbindungen die ausgesprochenen Merkmale lockerer Verbindungen. Ihre Bildung beim Zusammenschmelzen der Metalle verläuft nämlich ganz ruhig, und ist nach MuthmanN ${ }^{1}$ wahrscheinlich sogar endotherm, was betreffs der Bildung der Verbindung $\mathrm{Ce}_{4} \mathrm{Mg}$ im kristallisierten Zustande jetzt erwiesen ist. Ferner liegen ihre Schmelzpunkte durchweg bei niedrigen Temperaturen; besonders auffallend ist die tiefe Lage des Schmelzpunktes bei der endothermen Verbindung $\mathrm{Ce}_{4} \mathrm{Mg}$. Demgemäß sind die Maxima auf den Gleichgewichtskurven der Verbindungen sehr abgeflacht, und zwar in steigendem Maße mit dem zunehmenden Cer-Gehalt der Verbindungen. Hierdurch unterscheidet sich das Zustandsdiagramm der Cer-Magnesiumlegierungen von denen der genannten andern Cer-Legierungen in charakteristischer Weise.

Da also die Verbindungen des Cers mit Magnesium im Gegensatz zu den sehr festen Al-, Si-, Sn-, Pb-, Bi-Verbindungen nur locker sind, so muß, trotz des Vorhandenseins von Verbindungen, das Cer dem Magnesium verwandtschaftlich viel näher stehen, als den genannten Elementen, was bei der Ähnlichkeit in dem chemischen Charakter von Cer und Magnesium, welche sich z. B. in der großen Verbindungsfähigkeit bei höherer Temperatur mit fast allen übrigen Elementen, insbesondere mit dem Sauerstoff, in ihrer Fähigkeit kochendes Wasser zu zersetzen usw. äußert, auch nicht überraschend ist.

G. A. BARBIERI ${ }^{2}$ gelang es kürzlich, ein komplexes Cer-MolybdänSalz $4\left(\mathrm{NH}_{4}\right)_{2} \mathrm{O}-\mathrm{CeO}_{2}-12 \mathrm{MoO}_{3}-8 \mathrm{H}_{2} \mathrm{O}$ darzustellen, dessen Formel dem entsprechenden Cer-Thorium-Salz $4\left(\mathrm{NH}_{4}\right)_{2} \mathrm{O}-\mathrm{ThO}_{2}-12 \mathrm{MoO}_{3}-8 \mathrm{H}_{2} \mathrm{O}$ vollkommen analog ist. Der Umstand, da $B$ sich Cer und Thorium in diesen Salzen gleichwertig vertreten können und daß sich die Vierwertigkeit des Cers aus derartigen komplizierten Verbindungen ableiten läßt, scheint ihm ein Argument zu sein, das überzeugender für die Zugehörigkeit des Cers zur 4. Gruppe des periodischen Systems spricht, als die heftige Bildung sehr fester binärer Verbindungen des Cers mit Zinn und Blei dagegen. Gegenüber dieser

1 1. c.

1 Atti, R. Accad. dei Lincei, Rom, 28 I, (1914), 805. 
Auffassung muß man mit Tammans betonen, da $B$ sich die chemischen Beziehungen zweier Elemente zueinander bei der gegenseitigen Einwirkung der freien Elemente selbst, wobei der Einfluß fremder Elemente ausgeschlossen ist, am unmittelbarsten äußern müssen. Ein Beweis hierfür ist ja die überraschende Individualität der Valenz, welche sich in den Formeln binärer Verbindungen, nicht nur der Metalle unter sich, sondern auch in den Formeln der Oxyde, z. B. von Blei und Chrom, und der Sulfide, z. B. von Kalium, kundgibt. Dagegen kann der individuelle Affinitätscharakter der Elemente in den komplizierteren Verbindungen mehrerer Elemente sehr zurücktreten, wie uns die große Gleichartigkeit der Valenz, welche oft die verschiedenartigsten Elemente in ihren Salzverbindungen äußern, zeigt und wie auch aus dem Umstande hervorgeht, da $B$ ganz allgemein unter den komplizierteren Verbindungen die lockeren vorherrschen, während man umgekehrt unter den einfachen, binären Verbindungen sowohl sehr feste als auch sehr lockere findet.

Man sollte daher in der Frage nach der chemischen Verwandtschaft zweier Elemente nicht zu viel Gewicht legen auf die Übereinstimmung der Formeln; auf die Formeln bin ärer Verbindungen nicht, weil in ihnen selbst kleine Individualitätsuntersehiede Verschiedenheiten der Formeln bedingen können und auf kompliziertere Formeln nicht, weil in ihnen umgekehrt die Individualität einzelner Elemente im Molekül so sebx verwischt werden kann, daß selbst eine große Verschiedenartigkeit zweier darin enthaltener Elemente keine Verschiedenheit der Formeln hervorzurufen braucht.

Viel bedeutsamer ist die Fähigkeit zweier Elemente untereinander Verbindungen einzugehen, wie die Tammannsche Regel, wonach die Elemente einer natürlichen Gruppe im engeren Sinne untereinander keine Verbindungen eingehen, zeigt. Die Verbindungsfähigkeit kann sich aber, wie die Erfahrung gezeigt hat, unter Umständen auch schon bei so geringen Unterschieden des chemischen Charakters zeigen, wie sie unter den Gliedern einer natürlichen Gruppe von Elementen bestehen und die Ausnahmen von obiger Regel sprechen daher keineswegs gegen die Regel, sondern sind vielmehr nur ein Beweis für die individuelle Äußerung der Eigenschaften der Elemente in ihren binären Verbindungen.

Bei Anwendung der Tammannschen Regel betreffend die Verwandtschaft zweier Elemente hat man daher auch nicht bloß den Umstand zu berücksichtigen, daß Verbindungen vorhanden sind, sondern entscheidend ist vor allem die Festigkeit der Verbin- 
dungen, denn die Existenz von Verbindungen besagt nur, daß eine chemische Verschiedenheit zwischen den Elementen besteht, über den Grad dieser Versehiedenheit aber die Festigkeit der Verbindungen. So bilden zwar auch die einander nahe verwandten Halogene unter sich mehrere sehr lockere Verbindungen, niemals aber hat man beobachtet, daß nahe verwandte Elemente einer natürlichen Gruppe im engeren Sinne eine stark exotherme feste Verbindung untereinander eingehen.

Göttingen, Institut für physikalische Chemie der Universität.

Bei der Redaktion eingegangen am 6. Februar 1915. 